\title{
Originalien
}

Ophthalmologe 2020 · 117:1092-1099 https://doi.org/10.1007/s00347-020-01074-w Online publiziert: 5. März 2020

(c) Der/die Autor(en) 2020

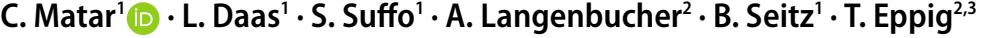

'Klinik für Augenheilkunde, Universitätsklinikum des Saarlandes UKS, Homburg/Saar, Deutschland ${ }^{2}$ Institut für Experimentelle Ophthalmologie, Universität des Saarlandes, Homburg/Saar, Deutschland ${ }^{3}$ AMIPLANT GmbH, Schnaittach, Deutschland

\section{Reliabilität der Hornhauttomographie nach Implantation von intrakornealen Ringsegmenten bei Keratokonus}

konus teilweise eine inflammatorische Ätiologie hat [11].

INTACS (Addition Technology Inc., Fremont, CA, USA), eine Art intrakornealer Ringsegmente (ICRS), bestehen aus bogenförmigen elliptischen Segmenten aus Polymethylmethacrylat (PMMA), die chirurgisch in das tiefe Hornhautstroma ( $80 \%$ Tiefe) des Auges eingeführt werden, um die Hornhaut mechanisch zu stabilisieren und die zentrale Hornhaut abzuflachen ([2]; - Abb. 1). Ursprünglich für die refraktive Korrektur von leichter Myopie konzipiert, gelten sie heute als fester Bestandteil der stadiengerechten Therapie des Keratokonus (KK).

Bereits 2001 berichteten Colin et al. zum ersten Mal über die Sicherheit und Wirksamkeit von INTACS nach Implantation bei 10 Keratokonuspatienten [5]. Seitdem hat dieses Verfahren an Beliebtheit bei refraktiven Chirurgen gewonnen. Für Keratokonuspatienten mit Kontaktlinsenintoleranz stellen INTACS die Alternative zu einer frühen Keratoplastik dar [7]. Weitere Indikationen sind Post-LASIK-Keratektasie und die pellucide marginale Degeneration [8]. Voraussetzungen für die Implantation sind das Fehlen von zentralen Hornhautnarben, eine Obergrenze des steilsten K-Wertes von nicht mehr als $58 \mathrm{dpt}$ und eine minimale Hornhautdicke von $400 \mu \mathrm{m}$ an der Implantationsstelle. Da die Ausdünnung bei der pelluciden marginalen Degeneration limbusnah liegt, ist die INTACS-Implantation mit dem Femtosekundenlaser aufgrund der Applanation bzw. des gekrümmten Interfaces deutlich erschwert.

Es ist bekannt, dass INTACS sowohl die unkorrigierte und die bestkorrigierte Sehschärfe verbessern und den Astigmatismus verringern sollen $[8,13]$. Zum anderen scheinen sie auch die Progression des $\mathrm{KK} z u$ hemmen. Bedi et al. publizierten 2012 refraktive und topographische postoperative Ergebnisse 5 Jahre nach konsekutiver fs-INTACSImplantation bei 105 Augen von KKPatienten [1]. Ferner wurde zwischen Augen mit dokumentierter präoperativer Progression (Zunahme im steilen Keratometriewert [Ks] um mehr als 1,00 dpt über 12 Monate präoperativ) und Augen ohne dokumentierte präoperative Progression unterschieden. Dabei zeigte sich insgesamt bei 91,3\% der Augen beider Gruppen und bei 92,9\% der Augen mit dokumentierter präoperativer Progression ein stabiler postoperativer Verlauf

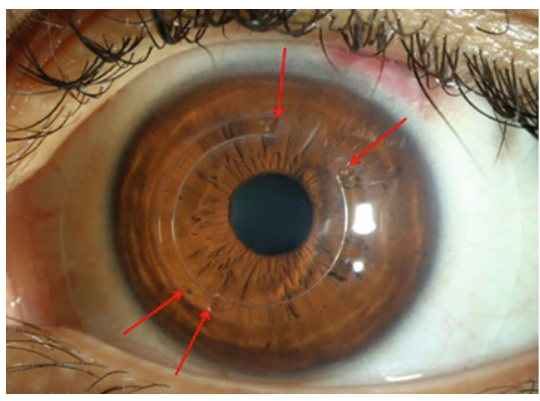

Abb. 1 \& Spaltlampenmikroskopische Aufnahme am ersten postoperativen Tag nach femtosekundenlasergestützten INTACS-Implantation (fs-INTACS, rote Pfeile) bei einem Keratokonusauge 
Tab. 1 Mittelwert \pm Standardabweichung aus den 5 Wiederholungsmessungen der eingeschlossenen Augen

\begin{tabular}{|c|c|c|c|c|c|c|c|c|c|c|}
\hline \multirow[t]{2}{*}{ ID } & \multicolumn{5}{|l|}{ Casia 2} & \multicolumn{5}{|c|}{ Pentacam HR } \\
\hline & Km & PKm & CCT & TCT & $K_{\text {MAX }}$ & Km & PKm & CCT & TCT & $K_{\text {MAX }}$ \\
\hline 1 & $46,09 \pm 0,18$ & $-8,19 \pm 0,04$ & $501 \pm 2$ & $465 \pm 2$ & $52,02 \pm 0,73$ & $46,48 \pm 0,08$ & $-7,30 \pm 0,10$ & $493 \pm 30$ & $476 \pm 32$ & $57,78 \pm 0,35$ \\
\hline 2 & $47,25 \pm 0,14$ & $-8,47 \pm 0,06$ & $481 \pm 2$ & $433 \pm 1$ & $53,15 \pm 0,74$ & $49,04 \pm 0,21$ & $-7,76 \pm 0,05$ & $483 \pm 4$ & $461 \pm 5$ & $60,94 \pm 1,97$ \\
\hline 3 & $45,70 \pm 0,17$ & $-7,56 \pm 0,06$ & $457 \pm 2$ & $449 \pm 1$ & $50,88 \pm 0,47$ & $46,62 \pm 0,08$ & $-7,54 \pm 0,11$ & $473 \pm 3$ & $469 \pm 4$ & $55,24 \pm 0,31$ \\
\hline 4 & $50,64 \pm 0,13$ & $-8,51 \pm 0,02$ & $433 \pm 1$ & $420 \pm 1$ & $56,77 \pm 0,24$ & $53,28 \pm 0,08$ & $-8,66 \pm 0,05$ & $439 \pm 5$ & $425 \pm 5$ & $62,34 \pm 0,43$ \\
\hline 5 & $43,93 \pm 0,14$ & $-7,18 \pm 0,04$ & $527 \pm 2$ & $487 \pm 4$ & $49,30 \pm 0,15$ & $44,06 \pm 0,17$ & $-6,68 \pm 0,39$ & $509 \pm 21$ & $478 \pm 28$ & $54,42 \pm 0,95$ \\
\hline 6 & $47,68 \pm 0,27$ & $-8,11 \pm 0,04$ & $437 \pm 4$ & $417 \pm 3$ & $53,86 \pm 0,78$ & $48,38 \pm 0,08$ & $-7,44 \pm 0,05$ & $442 \pm 9$ & $430 \pm 8$ & $56,08 \pm 1,01$ \\
\hline 7 & $50,86 \pm 0,19$ & $-8,25 \pm 0,06$ & $451 \pm 5$ & $413 \pm 5$ & $59,22 \pm 1,19$ & $51,14 \pm 0,26$ & $-7,46 \pm 0,05$ & $445 \pm 5$ & $429 \pm 5$ & $63,04 \pm 1,09$ \\
\hline 8 & $42,56 \pm 0,11$ & $-6,52 \pm 0,05$ & $471 \pm 2$ & $461 \pm 2$ & $49,16 \pm 0,20$ & $42,64 \pm 0,05$ & $-6,20 \pm 0,10$ & $488 \pm 5$ & $469 \pm 13$ & $48,70 \pm 1,19$ \\
\hline 9 & $44,34 \pm 0,05$ & $-7,14 \pm 0,10$ & $509 \pm 1$ & $474 \pm 2$ & $49,29 \pm 0,34$ & $43,90 \pm 0,20$ & $-6,58 \pm 0,04$ & $514 \pm 4$ & $498 \pm 3$ & $49,36 \pm 0,61$ \\
\hline 10 & $52,18 \pm 0,16$ & $-8,99 \pm 0,05$ & $424 \pm 2$ & $392 \pm 2$ & $66,35 \pm 0,63$ & $54,96 \pm 0,21$ & $-8,34 \pm 0,05$ & $433 \pm 4$ & $411 \pm 2$ & $74,56 \pm 0,46$ \\
\hline 11 & $47,86 \pm 0,27$ & $-7,77 \pm 0,08$ & $457 \pm 2$ & $409 \pm 0$ & $59,11 \pm 0,46$ & $47,16 \pm 0,55$ & $-6,72 \pm 0,16$ & $457 \pm 6$ & $431 \pm 1$ & $63,84 \pm 1,51$ \\
\hline 14 & $48,98 \pm 0,17$ & $-7,63 \pm 0,03$ & $474 \pm 1$ & $442 \pm 0$ & $58,92 \pm 0,39$ & $48,34 \pm 0,21$ & $-6,88 \pm 0,04$ & $471 \pm 2$ & $440 \pm 5$ & $63,66 \pm 0,36$ \\
\hline 15 & $51,14 \pm 0,25$ & $-8,67 \pm 0,08$ & $453 \pm 1$ & $417 \pm 1$ & $65,00 \pm 0,27$ & $51,54 \pm 0,67$ & $-8,36 \pm 0,11$ & $439 \pm 7$ & $419 \pm 8$ & $62,36 \pm 1,13$ \\
\hline 16 & $50,28 \pm 0,09$ & $-8,78 \pm 0,03$ & $333 \pm 1$ & $331 \pm 1$ & $60,18 \pm 0,13$ & $50,72 \pm 0,20$ & $-9,02 \pm 0,04$ & $329 \pm 4$ & $322 \pm 4$ & $59,98 \pm 0,66$ \\
\hline 17 & $39,81 \pm 0,57$ & $-7,13 \pm 0,26$ & $478 \pm 5$ & $453 \pm 2$ & $46,81 \pm 0,54$ & $40,54 \pm 0,15$ & $-6,80 \pm 0,14$ & $479 \pm 3$ & $470 \pm 2$ & $51,04 \pm 0,39$ \\
\hline 18 & $42,61 \pm 0,57$ & $-7,74 \pm 0,26$ & $451 \pm 9$ & $433 \pm 6$ & $54,98 \pm 1,05$ & $44,30 \pm 0,19$ & $-7,78 \pm 0,16$ & $456 \pm 4$ & $447 \pm 4$ & $57,76 \pm 0,43$ \\
\hline 19 & $47,01 \pm 0,08$ & $-7,74 \pm 0,06$ & $460 \pm 3$ & $430 \pm 1$ & $51,55 \pm 0,35$ & $46,80 \pm 0,14$ & $7,50 \pm 0,12$ & $469 \pm 2$ & $455 \pm 2$ & $53,62 \pm 0,34$ \\
\hline
\end{tabular}

Km mittlerer keratometrischer Brechwert der Hornhaut, $P K m$ mittlerer Brechwert der Hornhautrückfläche, CCT Hornhautdicke am Apex, TCT Hornhautdicke an der dünnsten Stelle, $K_{\max }$ keratometrischer Brechwert der Hornhaut an der steilsten Stelle

zwischen 1 und 5 Jahren postoperativ. Eine postoperative Progression wurde laut Autoren als Zunahme der Ks um mehr als 1 dpt über einen Zeitraum von 4 Jahren (zwischen 1 und 5 Jahren postoperativ) definiert. Zwar bislang noch nicht einheitlich definiert, spricht man laut Beschluss des gemeinsamen Bundesausschusses, die im Januar 2019 in Kraft trat, von einer Progression, wenn innerhalb von 12 Monaten eine Zunahme der maximalen Hornhautbrechkraft $\left(\mathrm{K}_{\max }\right)$ um mehr als $1 \mathrm{dpt}$ oder eine Zunahme des durch die subjektive Refraktion bestimmten Astigmatismus um $\geq 1 \mathrm{dpt}$ oder eine Abnahme der Basiskurve der bestsitzenden Kontaktlinse um $\geq 0,1 \mathrm{~mm}$ nachgewiesen werden kann [12]. In der Literatur wurden zahlreiche potenzielle Hornhautparameter zur Identifikation einer Progression beschrieben $[3,6]$. Um eine Aussage zur KK-Progression zu treffen, sind jedoch Kenntnisse über die Reproduzierbarkeit der Messverfahren unbedingt erforderlich. Unter Reproduzierbarkeit wird die Übereinstimmung von Messergebnissen verstanden, die unter (leicht) veränderten Bedingungen mit der gleichen Methode gemessen werden. Meist sind dies andere Umgebungsbe- dingungen, andere Positionierung des Patienten oder andere Untersucher.

Das Ziel dieser Arbeit war es, die Reproduzierbarkeit tomographischer Parameter in Augen mit KK, die sich einer femtosekundenlasergestützten INTACSImplantation (fs-INTACS) untergezogen hatten, vergleichend für 2 verschiedene Hornhauttomographiegeräte zu beurteilen.

\section{Studiendesign und Unter- suchungsmethoden}

Insgesamt wurden 19 Augen mit der Diagnose Keratokonus von 12 Patienten (Durchschnittsalter $33 \pm 10$ Jahre [20 bis 55 Jahre]) eingeschlossen. Alle Patienten hatten eine fs-INTACS-Implantation zwischen 2014 und 2018 durch 2 erfahrene Operateure aufgrund von $\mathrm{KK}$ von Stadium 2 oder größer (gemäß topographischer Keratokonusklassifikation [TKC]) erhalten. Bei allen Patienten wurde präoperativ die Hornhautdicke an der Implantationsstelle (6-mm-Zone) mit dem VA-OCT gemessen und der Tunnel mit dem Femtosekundenlaser kreiert, um die INTACS-Implantate in der optischen 6- bis 7-mm-Zone zu im- plantieren. Die Ringsegmente wurden immer tief im kornealen Stroma $(80 \%$ von der dünnsten Stelle der Hornhaut in der 6-mm-Zone) implantiert. Es wurden folgende Einstellungen am Femtosekundenlaser vorgenommen: Tiefe des Tunnels $80 \%$ der dünnsten Stelle der Hornhaut in der 6-mm-Zone, Innendurchmesser $6,0 \mathrm{~mm}$, Außendurchmesser $7,0 \mathrm{~mm}$, Laserenergie $1,5 \mathrm{~mJ}$. Die Dicke der Ringsegmente wurde entsprechend einem Nomogramm des Herstellers auf die individuelle Hornhaut abgestimmt. Bei allen Patienten wurde postoperativ eine Verbandskontaktlinse (AIR OPTIX ${ }^{\circledR}$ Night\&Day Aqua, Ciba Vision GmbH, Großwallstadt, Deutschland) 1 Woche lang aufgesetzt. Dazu wurden Prednisolon (Inflanefran forte ${ }^{\circledR}$ $10 \mathrm{mg} / \mathrm{ml}$, Allergan Pharmaceuticals Ltd., Irland) und Moxifloxacin Hydrochlorid 0,5\% (Vigamox, Alcon Pharma $\mathrm{GmbH}$, Freiburg/Breisgau, Deutschland) 6-mal täglich im Wechsel appliziert und die kortisonhaltigen Augentropfen anschließend stufenweise wöchentlich um 1 Tropfen reduziert. Konservierungsmittelfreie Befeuchtungstropfen (Optive UD Augentropfen; Allergan Pharmaceuticals) wurden zusätzlich bei allen 
Ophthalmologe 2020 - 117:1092-1099 https://doi.org/10.1007/s00347-020-01074-w

(c) Der/die Autor(en) 2020

\section{Matar $\cdot$ L. Daas $\cdot$ S. Suffo $\cdot$ A. Langenbucher $\cdot$ B. Seitz $\cdot$ T. Eppig}

\section{Reliabilität der Hornhauttomographie nach Implantation von intrakornealen Ringsegmenten bei Keratokonus}

\author{
Zusammenfassung \\ Hintergrund und Ziel. Intrakorneale Ringseg- \\ mente (ICRS) sollen die Progression des \\ Keratokonus (KK) aufhalten. Diese Aussage zur \\ Progression erfordert jedoch Kenntnisse über \\ die Reproduzierbarkeit der angewendeten \\ Messverfahren. Ziel dieser Studie war es, \\ die Reproduzierbarkeit tomographischer \\ Parameter in Augen mit Keratokonus \\ (KK) nach femtosekundenlasergestützter \\ INTACS-Implantation (fs-INTACS) zwischen \\ 2 Hornhauttomographen zu vergleichen. \\ Patienten und Methoden. 19 KK-Augen \\ wurden eingeschlossen. 5 Messungen \\ wurden mit dem Scheimpflug-Tomogra- \\ phen Pentacam HR und dem optischen \\ Kohärenztomographen (VA-OCT) Casia 2 \\ durchgeführt. Zielgrößen waren die
}

Reproduzierbarkeit und die Vergleichbarkeit der Messungen von (1) keratometrischem Brechwert der Hornhautvorder- und (2) -rückfläche, (3) maximalem keratometrischem Brechwert, (4) zentraler Hornhautdicke und (5) Hornhautdicke an der dünnsten Stelle zwischen beiden Geräten.

Ergebnisse. Die mittlere Differenz (Pentacam minus VA-OCT) von (1), (2), (3), (4) und (5) lag bei $0,67 \mathrm{dpt}, 0,41 \mathrm{dpt}, 3,4 \mathrm{dpt}$, $1,5 \mu \mathrm{m}$ und $11,8 \mu \mathrm{m}$. Die mittlere SD der 5 Wiederholungen für (1), (2), (3), (4) und (5) lag bei $0,20 \mathrm{dpt} / 0,20 \mathrm{dpt}, 0,10 \mathrm{dpt} / 0,07 \mathrm{dpt}$, $0,75 \mathrm{dpt} / 0,5 \mathrm{dpt}, 6,5 / 2,4 \mu \mathrm{m}(p=0,007)$ und $7,3 \mu \mathrm{m} / 1,9 \mu \mathrm{m}(p=0,001)$ für Pentacam/Casia 2. Cronbach's a war für alle Geräte und Parameter besser als 0,98.
Schlussfolgerung. Casia 2 und Pentacam ermöglichen beide eine zuverlässige Beurteilung der Hornhautbrechkraft bei KK nach fsINTACS-Implantation. Die Reproduzierbarkeit war nur für die Hornhautdickenmessung mit Casia 2 signifikant besser. Pentacam zeigte signifikant höhere Werte für die Brechkraft der Hornhautvorder- und Rückfläche und misst signifikant dicker an der dünnsten Stelle im Vergleich zu Casia 2.

\section{Schlüsselwörter}

Keratokonus · INTACS · Pentacam ·

Vorderaugenabschnitts-OCT · Casia 2 .

Reproduzierbarkeit

\section{Reliability of corneal tomography after implantation of intracorneal ring segments for keratoconus}

\section{Abstract}

Background and objective. Intracorneal ring segments (ICRS) are believed to stop the progression of keratoconus (KC). This statement on progression, however, requires knowledge about measurement reproducibility. The purpose of this study was to compare the reproducibility of tomographic parameters in eyes with KC after femtosecond laser-assisted implantation of INTACS (fs-INTACS) using two different devices and to determine which is more reliable for the follow-up of these patients.

Patients and methods. In this study $19 \mathrm{KC}$ eyes were included and repeatedly examined 5 times with the Scheimpflug topography Pentacam HR and the Casia 2 optical coherence tomography (VA-OCT) devices. Outcome measures included the reproducibility and comparability of measurements between the two devices of (1) keratometric refractive power of the anterior cornea and (2) posterior cornea, (3) maximum keratometric refractive power, (4) central corneal thickness and (5) corneal thickness at the thinnest site. Results. The mean differences (Pentacam minus Casia 2) of (1), (2), (3), (4) and (5) were $0.67 \mathrm{dpt}, 0.41 \mathrm{dpt}, 3.4 \mathrm{dpt}, 1.5 \mu \mathrm{m}$ and $11.8 \mu \mathrm{m}$, respectively. The mean SDs of the 5 repeat measurements for (1), (2), (3), (4) and (5) were $0.20 \mathrm{dpt} / 0.20 \mathrm{dpt}, 0.10 \mathrm{dpt} / 0.07 \mathrm{dpt}$, $0.75 \mathrm{dpt} / 0.5 \mathrm{dpt}, 6.5 \mu \mathrm{m} / 2.4 \mu \mathrm{m}(p=0.007)$ and $7.3 \mu \mathrm{m} / 1.9 \mu \mathrm{m}(p=0.001)$ for Pentacam and Casia 2, respectively. Cronbach's alpha was better than 0.98 for both devices and all parameters.

Conclusion. Both Casia 2 and Pentacam enable a reliable assessment of the corneal refractive power in KC after fs-INTACS implantation; however, the reproducibility was significantly better with Casia 2 only for the measurement of corneal thickness. The Pentacam showed significantly higher values for the mean anterior and posterior corneal refractive power and measured significantly thicker at the thinnest point of the cornea compared to Casia 2.

\section{Keywords}

Keratoconus · INTACS · Pentacam · Anterior Segment-OCT $\cdot$ Casia $2 \cdot$ Reproducibility
Patienten initial 6-mal täglich angesetzt. Es wurden jeweils 5 Wiederholungsmessungen mit dem Scheimpflug-Tomographen Pentacam HR (Oculus Optikgeräte GmbH, Wetzlar, Deutschland) und dem optischen Kohärenztomographen des vorderen Augenabschnitts (VA-OCT) Casia 2 (Tomey Corp., Nagoya, Japan) durchgeführt.

Alle Messungen wurden am gleichen Tag mit einer Pause von 5 min zwischen den einzelnen Messungen unter mesopischen Bedingungen, bei spielender $\mathrm{Pu}$ - pille und durch denselben Untersucher durchgeführt. Vorab wurden die Patienten ausführlich über die korrekte Positionierung des Kopfes auf die Kinnstütze sowie über den geraden Augenblick und Fixierung des Zielobjekts im Zentrum der Aufnahmekamera während der einzelnen Messungen aufgeklärt. Die Untersuchungen wurden gemäß den ethischen Grundsätzen der Deklaration von Helsinki durchgeführt.

Primäre Zielgrößen waren die Reproduzierbarkeit von (1) mittlerem kerato- metrischem Brechwert der Hornhautvorderfläche (Km), (2) mittlerem Brechwert der Hornhautrückfläche (PKm), (3) maximalem keratometrischem Brechwert $\left(K_{\max }\right),(4)$ zentraler Hornhautdicke (CCT) und (5) Hornhautdicke an der dünnsten Stelle (TCT).

\section{Statistische Analyse}

Die erhobenen Werte wurden mittels SPSS ${ }^{\circledR}$ Version 24.0 (IBM, Armonk, USA) statistisch ausgewertet. Die Reproduzier- 


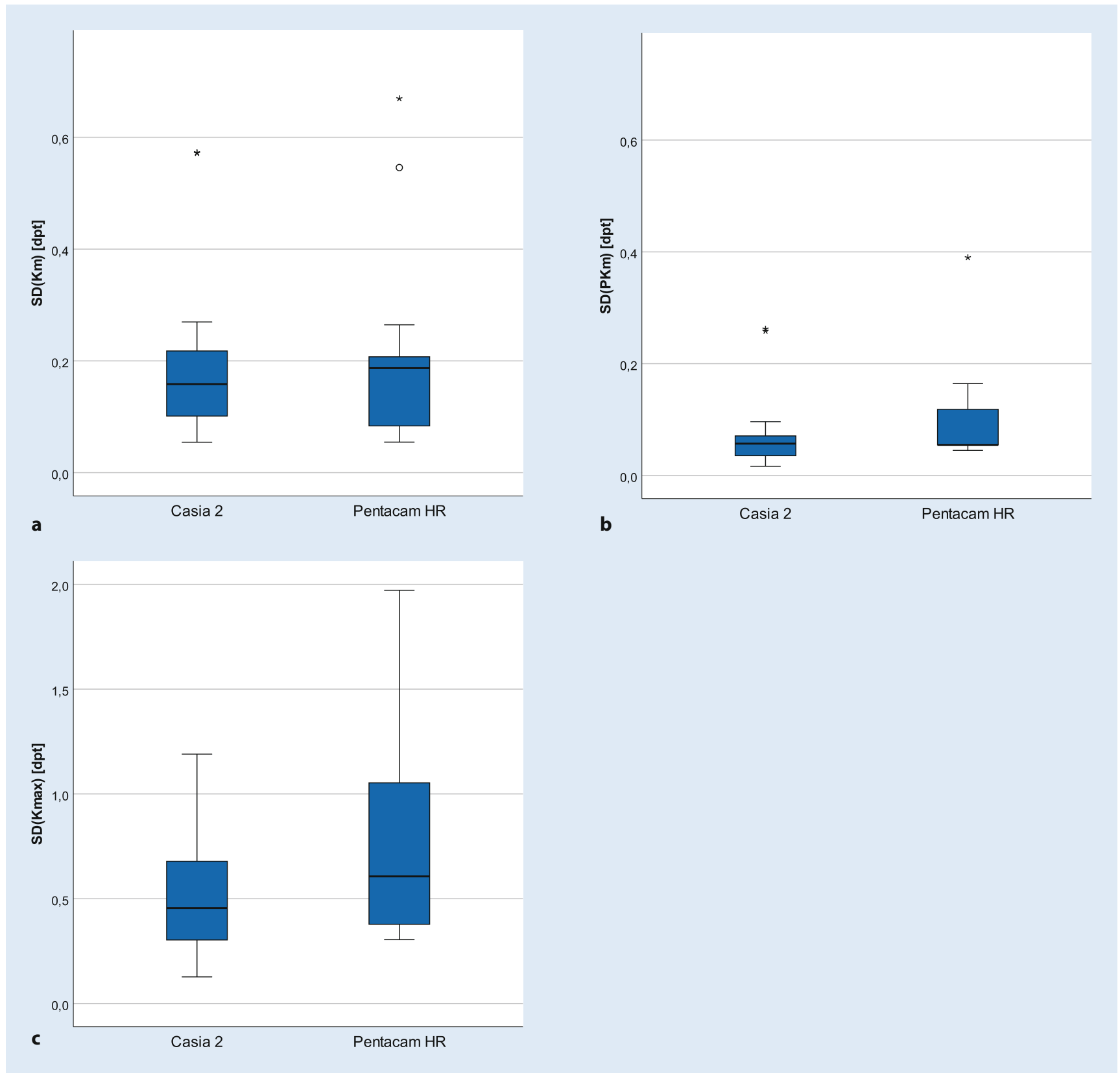

Abb. 2 ॥ Standardabweichung der vorderen (a) und hinteren (b) mittleren Brechwerte für beide Geräte im Vergleich sowie der maximale keratometrische Brechwert $\mathrm{K}_{\max }(\mathbf{c})$

barkeit wurde als mittlere Standardabweichung (SD) zwischen den 5 Wiederholungsmessungen ausgedrückt. Der Unterschied in der Reproduzierbarkeit wurde mit einem paarigen WilcoxonRangsummentest untersucht. $p$-Werte $<0,05$ wurden dabei als statistisch signifikant gewertet. Eine A-priori-Fallzahlanalyse mit der Software $G^{*}$ Power (Erdfelder, Faul, \& Buchner, HHU Düsseldorf, Deutschland) [10] ergab, dass für den Nachweis einer Effektgröße $d z=1$ (entsprechend 0,25 dpt Unterschied in der Keratometrie) und einer geforderten Power von $95 \%$ eine Fallzahl von 16 Fällen notwendig ist. Als Maß für die Reliabilität wurde zudem Cronbach's $\alpha$ berechnet. Die Vergleichbarkeit beider Geräte wurde anhand Bland-AltmannPlots dargestellt.

\section{Ergebnisse}

Die Anzahl der Augen in den TKC-Gruppen $2|3| 4$ betrug $4|8| 7$. Bei 4 Augen wurde 1 Ringsegment und bei 15 Augen wurden 2 Ringsegmente implantiert. Der zeitliche Abstand zwischen der fs-INTACSImplantation und der Messung betrug im Mittel 2,3 Jahre. Die Dicke des dickeren Ringsegments lag zwischen 300 und $450 \mu \mathrm{m}$. 


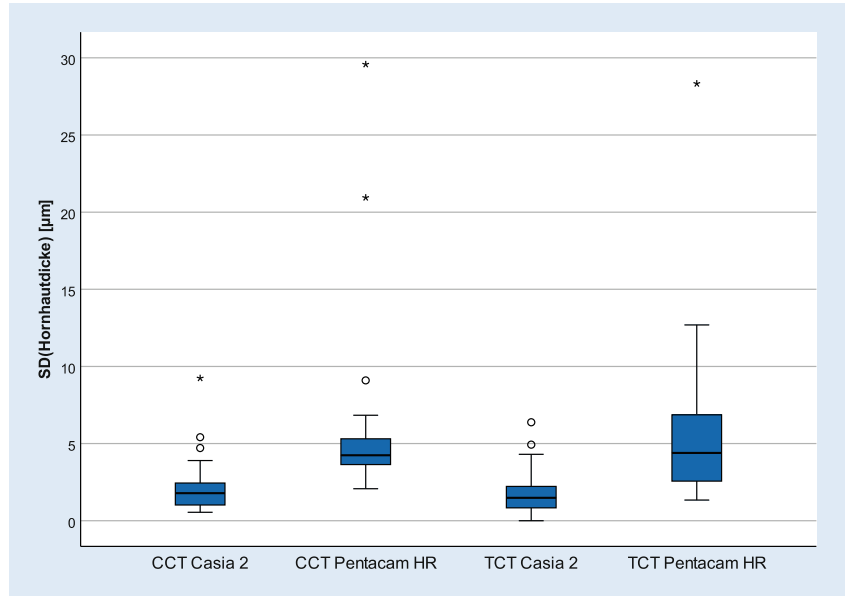

Abb. $3<$ Standardabweichung für die Messung der Hornhautdicke am Apex (zentrale Hornhautdicke [CCT]) und an der dünnsten Stelle (TCT) für beide Geräte im Vergleich

Die mittleren Messwerte aus den 5 Wiederholungsmessungen der einzelnen Augen und Geräte sowie die dazugehörige Standardabweichung sind in - Tab. 1 aufgeführt.

Die mittlere Standardabweichung (SD) des durchschnittlichen keratometrischen Hornhautbrechwerts $(\mathrm{Km})$ betrug bei der Pentacam 0,20 und beim Casia 2 $0,20 \mathrm{dpt}(p=0,629)(\bullet$ Abb. 2a). Der posteriore Hornhautbrechwert (PKm) zeigte eine mittlere SD von 0,10 vs. $0,07 \mathrm{dpt}$ $(p=0,117)$ im Vergleich von Pentacam zu Casia 2 (• Abb. 2b). Die mittlere SD des maximalen keratometrischen Hornhautbrechwerts $\left(K_{\max }\right)$ lag bei $0,75 \mathrm{dpt}$ bzw. 0,50 dpt für Pentacam und Casia 2 $(p=0,099)$. Die zentrale (CCT) und die dünnste Hornhautstelle (TCT) zeigten in der Pentacam im Vergleich zu Casia 2 eine mittlere SD von 6,5 vs. $2,4 \mu \mathrm{m}$ $(p=0,007)$ und 7,3 vs. $1,9 \mu \mathrm{m}(p=0,001)$

(- Abb. 3). Cronbach's a war für beide Geräte und Parameter besser als 0,98.

Die mittlere Differenz des anterioren bzw. posterioren mittleren Hornhautbrechwerts sowie des maximalen Keratometriewerts $K_{\max }$ zwischen beiden Geräten (Pentacam und Casia 2) lag bei $0,67 \pm 1,03 \mathrm{dpt}(p=0,02), 0,41 \pm 0,37 \mathrm{dpt}$ $(p=0,001)$ und $3,40 \pm 2,82 \mathrm{dpt}(p=0,01)$. Die mittlere Differenz der CCT und TCT lag bei $1,5 \pm 9 \mu \mathrm{m}(p=0,396)$ und $11,8 \pm 10,8 \mu \mathrm{m}$. Die Hornhautdicke an der dünnsten Stelle (TCT) wurde mit dem VA-OCT signifikant dünner gemessen als mit der Pentacam $(p=0,01)$. Die Grenzen der Übereinstimmung (BlandAltmann) für die Hornhautbrechwerte sind in $\bullet$ Abb. $4 a-c$, die für zentrale und dünnste Hornhautdicke in - Abb. 5a, b dargestellt.

\section{Diskussion}

Die Erkennung von Veränderungen eines Zustands mithilfe von Messmethoden kann nur dann gelingen, wenn die Messung hinreichend genau und reproduzierbar ist. Daher kann eine Progression eines Keratokonus nur dann sicher festgestellt werden, wenn die verwendeten Messmethoden eine höhere Reproduzierbarkeit aufweisen als der Grenzwert, der für eine Progression spricht.

Der Begriff der Reproduzierbarkeit („reproducibility“) wird oft mit dem Begriff der Wiederholbarkeit (,repeatability“) gleichgesetzt, jedoch unterscheiden diese sich in einem wesentlichen Punkt: Die Reproduzierbarkeit kann nur unter leicht geänderten Bedingungen ermittelt werden [14]. Dies kann z. B. eine andere Positionierung des Patienten bedeuten, ein anderes Messgerät des gleichen Typs oder ein anderer Untersucher. In unserer Studie wurde zwischen den einzelnen Messungen jeweils die Position des Patienten in der Kinnstütze verändert. Zudem wurde eine Zeitspanne zwischen den 5 Untersuchungen eingehalten, die mindestens das 5-Fache der Untersuchungsdauer beträgt (1 min Untersuchung, dann 5 min Pause, danach 1 min Untersuchung etc.).

Unsere Ergebnisse zeigen, dass beide Messgeräte bezüglich der mittleren Hornhautbrechwerte bei INTACS-Patienten eine hohe Reproduzierbarkeit von etwa 0,2 dpt aufweisen. Chen und Lam untersuchten die Reproduzierbarkeit von Pentacam-Messungen an Normalaugen zu 2 verschiedenen Zeitpunkten [4]. Vergleicht man diesen Wert mit dem "coefficient of repeatability" (1,96-fache Standardabweichung) aus der Studie von Chen und Lam [4], so erreichen beide Geräte bei Augen nach INTACS-Implantation eine etwa halbierte Reproduzierbarkeit im Vergleich zu Normalaugen $( \pm 0,39 \mathrm{dpt}$ im Vergleich $\mathrm{zu} \pm 0,21 \mathrm{dpt}$ für $\mathrm{Km}$ ). Hierdurch könnten wiederholt nachweisbare Veränderungen $\geq 0,5 \mathrm{dpt}$ als Progressionsverdacht gewertet werden. Allerdings gilt $\mathrm{zu}$ beachten, dass Kontaktlinsen und diverse Medikamente Veränderungen an der Hornhaut bewirken können, die eine Progression vortäuschen können. Veränderungen von $\geq 1 \mathrm{dpt}$, wie in den aktuellen Leitlinien empfohlen, sind jedoch bei wiederholter Messung als Progression definierbar. Der maximale Hornhautbrechwert $\mathrm{K}_{\max }$ zeigt eine deutlich größere Unsicherheit von $0,5 \mathrm{dpt}$ beim VA-OCT und 0,75 dpt bei der Pentacam. Dieser Parameter wird üblicherweise zur Definition einer Progression herangezogen, wobei eine Veränderung von $>1 \mathrm{dpt}$ als Progression gewertet wird [12]. Bezieht man sich wieder auf den „coefficient of repeatability“ nach Chen und Lam [4], so wäre mit VA-OCT der Grenzwert zur Feststellung der Progression von $1 \mathrm{dpt}$ anwendbar, im Falle der Pentacam müsste der Grenzwert jedoch auf bis $\mathrm{zu}$ 1,5 dpt angehoben werden. In jedem Fall empfiehlt es sich, die Aussage durch Wiederholung der Messung zu bestätigen [4]. Ebenfalls sehr deutlich fällt der Unterschied zwischen beiden Geräten bezüglich $\mathrm{K}_{\max }$ mit rund 3,4 dpt aus, der vermutlich durch die unterschiedlichen Messverfahren und ggf. fehlerhafte Erkennung der Hornhautfläche (z. B. durch erhöhte Streuung in der Umgebung des Implantats bei der Pentacam) begründet ist.

Bezüglich der Hornhautdicke zeigt sich ein differenzierteres Bild, das VAOCT weist hier eine höhere Reproduzierbarkeit nur bezüglich der Messung der Hornhautdicke auf. Die Bland-Altmann-Analyse zur Übereinstimmung zwischen beiden Geräten verdeutlicht, dass die Unterschiede zwischen beiden 

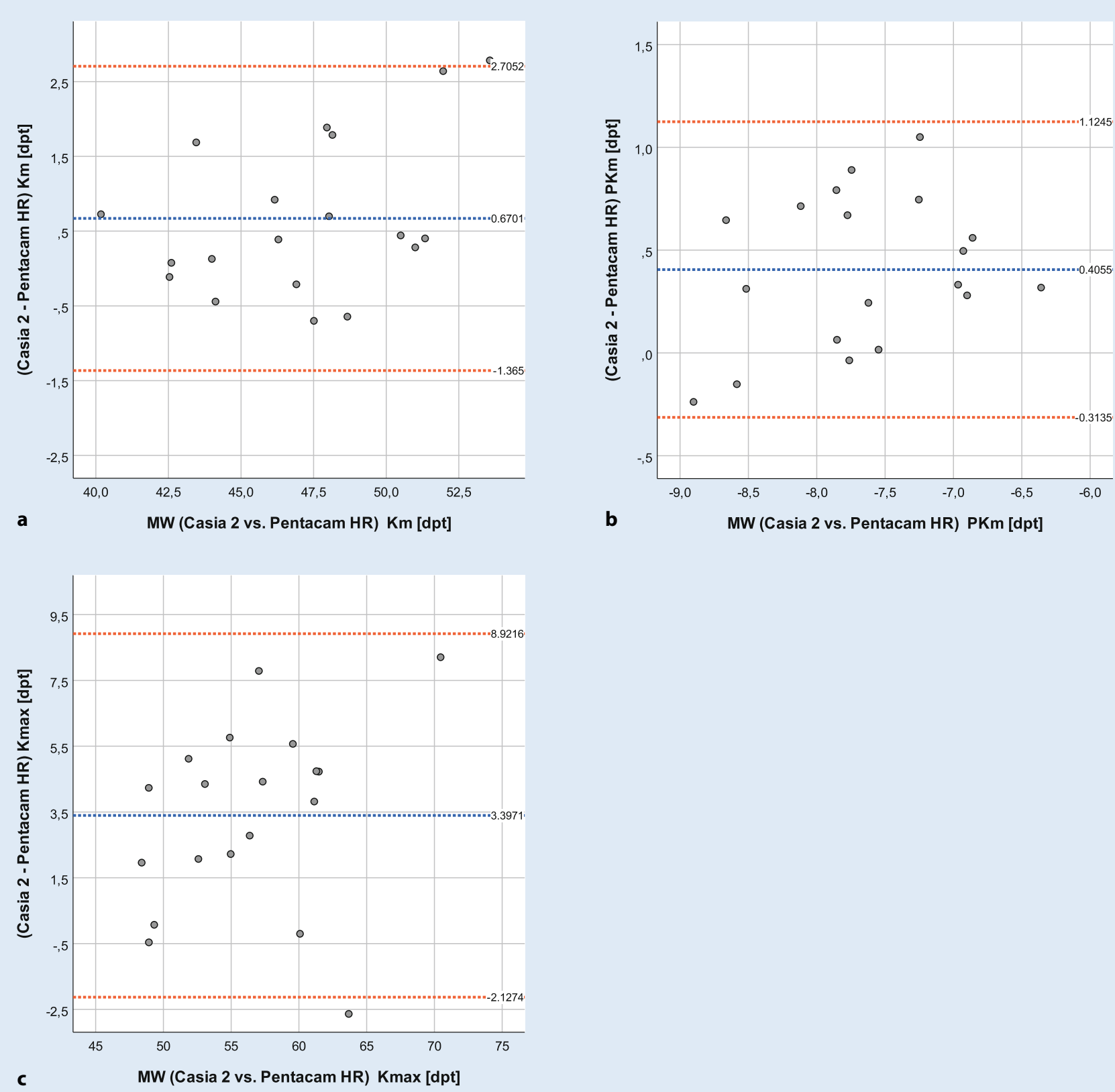

Abb. 4 ॥ Grenzen der Übereinstimmung bezüglich Mittlerwerten (MW) durchnittlicher anteriorer (a), posteriorer (b) und maximaler keratometrischer (c) Hornhautbrechwerte im Vergleich zwischen Pentacam HR und Casia 2. Dargestellt sind Mittelwert (blau) sowie die $\pm 1,96$-fache Standardabweichung (rot)

Geräten vergleichsweise groß ausfallen (mehrere Dioptrien bezüglich der Hornhautbrechung bzw. bis zu $20 \mu \mathrm{m}$ bezüglich der Hornhautdicke). Die Pentacam zeigte signifikant höhere Hornhautbrechwerte als das Casia 2. Zudem misst die Pentacam die Hornhautdicke an der dünnsten Stelle signifikant dicker. Demnach können beide Geräte nicht als gegeneinander austauschbar betrachtet werden. Eine analoge Auswertung unse- rer Arbeitsgruppe an Keratokonusaugen ohne ICRS zeigte, dass die Standardabweichung bei wiederholter Messung mit zunehmendem TKC-Stadium zunimmt [17]. Außerdem wurde die Dicke mittels VA-OCT um 10-30 $\mu \mathrm{m}$ stadienabhängig dünner gemessen [17]. Eine Arbeit von Wonneberger et al. kam zu dem Schluss, dass die Streuung des $\mathrm{K}_{\max }$-Wertes bei zunehmendem Keratokonusschweregrad ebenfalls zunimmt [18].
Schröder et al. untersuchten Pentacam HR und Casia SS-1000 in einer Serie von gesunden Augen in Bezug auf die kornealen Höhendaten und die $\mathrm{Pa}$ chymetrie [16]. Sie stellten fest, dass die Pentacam HR hier die größte Reproduzierbarkeit aufwies, gefolgt vom Casia SS1000. Die Auswertung bezog sich jedoch nicht auf die keratometrischen Brechwerte und ist daher schwer mit unserer Studie vergleichbar. In Übereinstimmung mit 

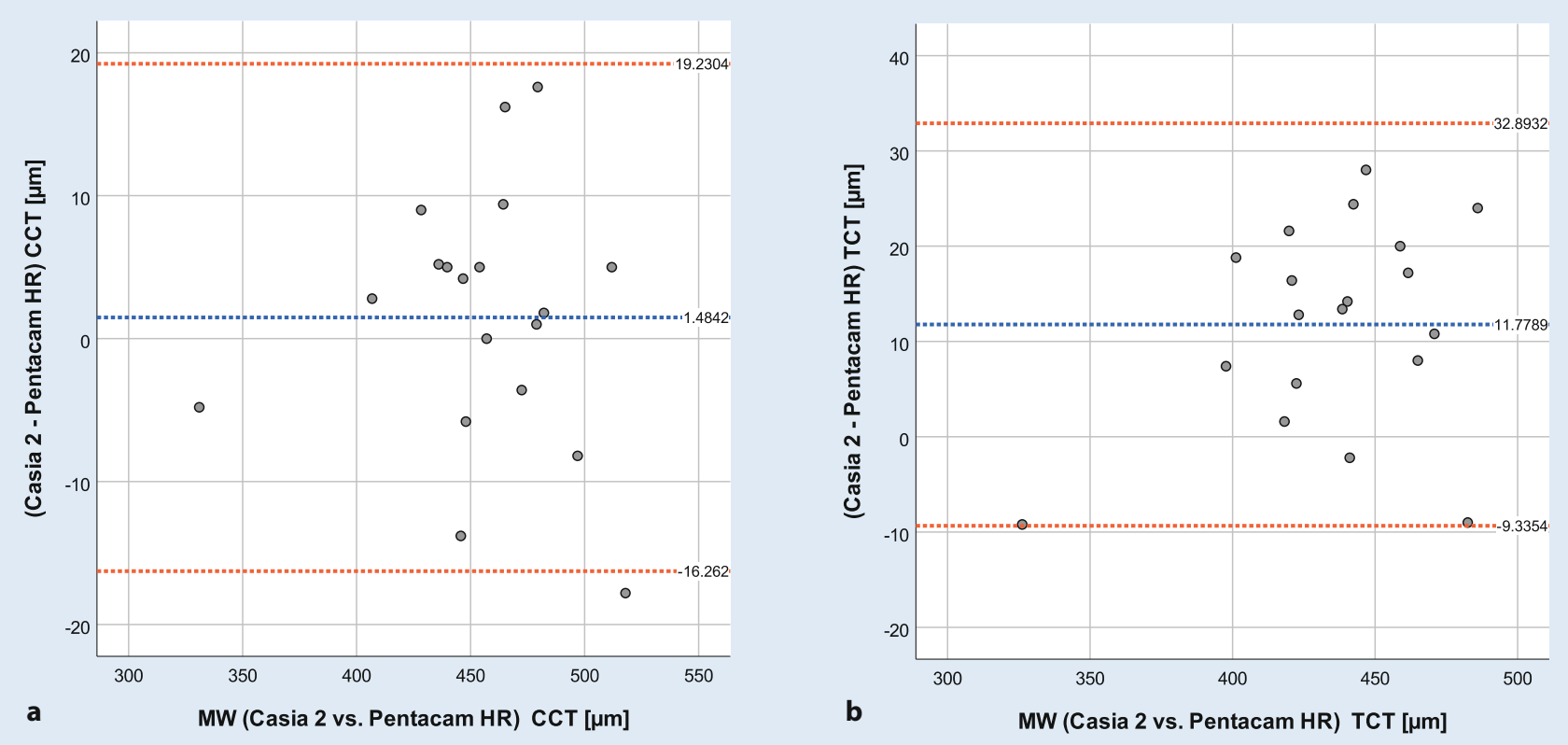

Abb. 5 ॥ Grenzen der Übereinstimmung bezüglich Mittlerwerten (MW) zentraler Hornhautdicke (CCT) (a) und Hornhautdicke an der dünnsten Stelle (TCT) (b) im Vergleich zwischen Pentacam HR und Casia 2. Dargestellt sind Mittelwert (blau) sowie die $\pm 1,96$-fache Standardabweichung (rot)

unserer Studie attestierten sie dem Casia SS-1000 eine höhere Reproduzierbarkeit bezüglich der Hornhautdickenmessung als der Pentacam HR.

Obwohl die Ultraschallpachymetrie immer noch als Goldstandard für die Hornhautdickenmessung gilt, handelt es sich um eine Kontaktmethode die mit einem erhöhten Risiko für Hornhautverletzungen und Infektionen insbesondere bei ektatischen Hornhäuten einhergeht. Inzwischen gilt die optische Messung der Hornhautdicke mehr und mehr als Standard. Diese erlaubt nicht nur, die Diagnose Keratokonus zu stellen bzw. die Kontraindikationen für die einzelnen therapeutischen Möglichkeiten zu erfassen (v.A. Crosslinking oder INTACS-Implantation), sondern auch den Verlauf präzise zu dokumentieren, um ggf. eine Eskalierung in der Therapie im Fall einer Progression zu rechtfertigen. Mehrere Studien verglichen die Hornhautdicke bei Augen mit Keratokonus mit verschiedenen Messgeräten. Bis jetzt ist uns jedoch keine Studie bekannt, die die Reproduzierbarkeit und Vergleichbarkeit der Hornhautdickenmessung und Keratometrie bei Keratokonusaugen mit INTACS-Implantaten zwischen 2 Tomographen untersuchte.

\section{Schlussfolgerung}

Wir schlussfolgern daraus, dass zwar das VA-OCT Casia 2 und die Pentacam aufgrund der gegebenen Reproduzierbarkeit der Messwerte für die Verlaufsbetrachtung nach INTACS-Implantation geeignet sind, die Messwerte jedoch nicht gegeneinander ausgetauscht werden dürfen. Für eine Progressionsbetrachtung sollte daher immer das gleiche Gerät verwendet werden.

\section{Korrespondenzadresse}

\section{Matar}

Klinik für Augenheilkunde, Universitätsklinikum des Saarlandes UKS

Kirrberger Str. 100, 66421 Homburg/Saar,

Deutschland

cedmatar@gmail.com

Funding. Open Access funding provided by Projekt DEAL.

\section{Einhaltung ethischer Richtlinien}

Interessenkonflikt. C. Matar, L. Daas, S. Suffo, A. Langenbucher, B. Seitz und T. Eppig geben an, dass kein Interessenkonflikt besteht.
Für diesen Beitrag wurden von den Autoren keine Studien an Menschen oder Tieren durchgeführt. Für die aufgeführten Studien gelten die jeweils dort angegebenen ethischen Richtlinien.

Open Access. Dieser Artikel wird unter der Creative Commons Namensnennung 4.0 International Lizenz veröffentlicht, welche die Nutzung, Vervielfältigung, Bearbeitung, Verbreitung und Wiedergabe in jeglichem Medium und Format erlaubt, sofern Sie den/die ursprünglichen Autor(en) und die Quelle ordnungsgemäß nennen, einen Link zur Creative Commons Lizenz beifügen und angeben, ob Änderungen vorgenommen wurden.

Die in diesem Artikel enthaltenen Bilder und sonstiges Drittmaterial unterliegen ebenfalls der genannten Creative Commons Lizenz, sofern sich aus der Abbildungslegende nichts anderes ergibt. Sofern das betreffende Material nicht unter der genannten Creative Commons Lizenz steht und die betreffende Handlung nicht nach gesetzlichen Vorschriften erlaubt ist, ist für die oben aufgeführten Weiterverwendungen des Materials die Einwilligung des jeweiligen Rechteinhabers einzuholen.

Weitere Details zur Lizenz entnehmen Sie bitte der Lizenzinformation auf http://creativecommons.org/ licenses/by/4.0/deed.de.

\section{Literatur}

1. Bedi R, Touboul D, Pinsard L, Colin J (2012) Refractive and topographic stability of Intacs in eyes with progressive keratoconus: five-year follow-up. JRefract Surg 28:392-396

2. Belin MW, Meyer JJ, Duncan JK, Gelman R, Borgstrom M (2017) Assessing progression of keratoconus and cross-linking efficacy: the 
Belin ABCD Progression Display. Int J Kerat Ect Cor Dis 6:1-10

3. Chatzis N, Hafezi F (2012) Progression of keratoconus and efficacy of pediatric corrected corneal collagen cross-linking in children and adolescents. JRefract Surg 28:753-758

4. Chen D, Lam AKC (2009) Reliability and repeatability of the Pentacam on corneal curvatures. Clin Exp Optom 92:110-118

5. Colin J, Cochener B, Savary G, Malet F (2000) Correcting keratoconus with intracorneal rings. J Cataract Refract Surg 26:1117-1122

6. Duncan JK, Belin MW, Borgstrom M (2016) Assessing progression of keratoconus: novel tomographic determinants. Eye Vis 3:6

7. El-Husseiny M, Tsintarakis T, Eppig T, Langenbucher A, Seitz B (2013) Intrakorneale Ringsegmente beim Keratokonus. Ophthalmologe 110:823-829

8. El-Husseiny M, Daas L, Langenbucher A, Seitz B (2016) Intrakorneale Ringsegmente bei Keratektasien - Zwischenergebnisse und potenzielle Komplikationen. Klin Monatsbl Augenheilkd 233:722-726

9. Eppig T, Spira-Eppig C, Goebels S, Seitz B, El-Husseiny M, Lenhart M, Papavasileiou K, Szentmáry N, Langenbucher A (2018) Asymmetry between leftand righteyesinkeratoconus patients increases with the severity of the worse eye. Curr Eye Res 43:848-855

10. Faul F, Erdfelder E, Buchner A, Lang A-G (2009) Statistical power analyses using $G^{*}$ Power 3.1: tests for correlation and regression analyses. Behav Res Methods 41:1149-1160

11. Galvis V, Sherwin T, Tello A, Merayo J, Barrera R, Acera A (2015) Keratoconus: an inflammatory disorder? Eye 29:843-859

12. https://www.g-ba.de/downloads/39-2613417/2018-07-19_MVV-RL_UV-VernetzungRiboflavin-Keratokonus_BAnz.pdf. Zugegriffen: 11. Okt. 2018

13. Hellstedt T, Mäkelä J, Uusitalo R, Emre S, Uusitalo R (2005) Treating keratoconus with intacs corneal ring segments. J Refract Surg 21:236-246

14. Plesser HE (2017) Reproducibility vs. replicability: a brief history of a confused terminology. Front Neuroinform 11:76

15. Rabinowitz YS (2013) INTACS for keratoconus and ectasia after LASIK. Int Ophthalmol Clin 53:27-39

16. Schröder S, Mäurer S, Eppig T, Seitz B, Rubly K, Langenbucher A (2018) Comparison of corneal tomography: repeatability, precision, misalignment mean elevation, and mean pachymetry. Curr Eye Res 43:709-716

17. Seitz B, Elzer B, Spira-Eppig C, Langenbucher A, Zemova E, Szentmáry N, Eppig T (2018) Repeatability of Scheimpflug imaging and anterior segment optical coherence tomography parameters in keratoconus is decreasing with increasing severity. Invest Ophthalmol Vis Sci 59:3436

18. Wonneberger W, Sterner B, MacLean U, Claesson M, Zetterberg M (2018) Repeated same-day versus single tomography measurements of keratoconic eyes for analysis of disease progression. Cornea 37:474-479

\section{In eigener Sache}

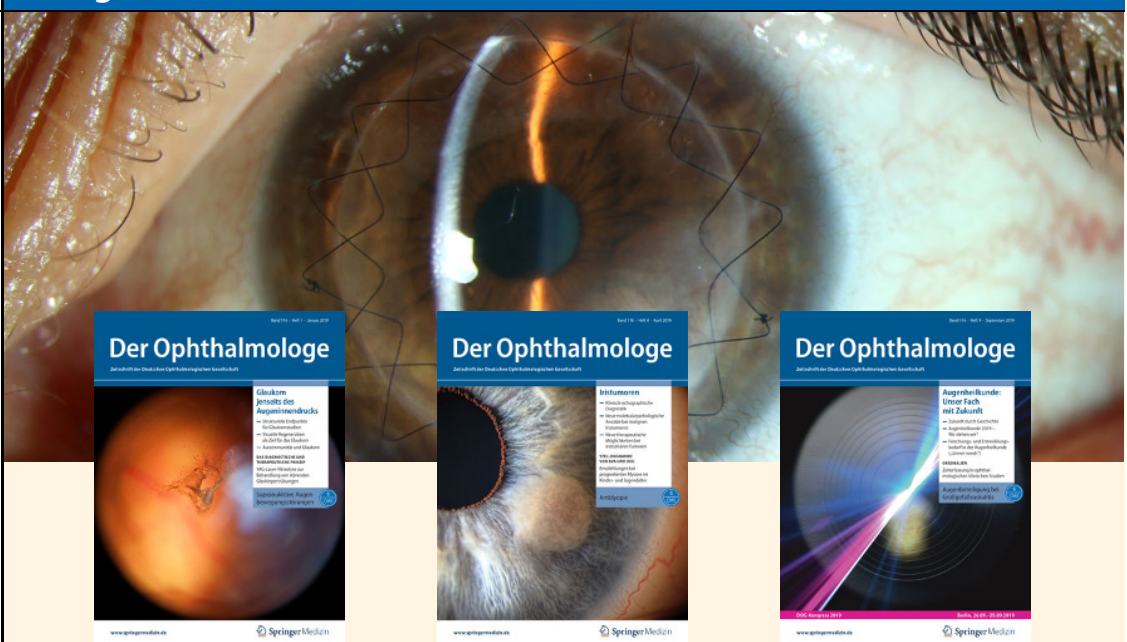

Leitthemenübersicht von Der Ophthalmologe

Der Ophthalmologe bietet Ihnen jeden Monat umfassende und aktuelle Beiträge zu interessanten Themenschwerpunkten aus allen Bereichen der Augenheilkunde.

Rückblick - 2019

01/19 Glaukom jenseits des

Augeninnendrucks

02/19 Neurotrophe Keratopathie

03/19 Therapie der Fuchs-Endothel-

dystrophie mittels DMEK

04/19 Iristumoren

05/19 Komplexe Glaukome mit hohem Behandlungsrisiko

06/19 Myopie-Epidemiologie,

Pathomechanismen und Prävention

07/19 Originalien

08/19 OCT-Angiographie und systemische

Erkrankungen

09/19 Augenheilkunde: unser Fach mit

Zukunft

10/19 Schwere Flüssigkeiten in der

Netzhautchirurgie

11/19 Makulaformen und andere

Makulopathien

12/19 Augenverletzungen durch

Feuerwerks- und Knallkörper

SpringerMedizin.de bietet Ihnen Zugang zu allen elektronisch verfügbaren Ausgaben Ihrer Zeitschrift - unabhängig davon, seit wann Sie die Zeitschrift abonniert haben.

\section{Rückblick \& Vorschau - 2020}

01/20 Originalien

02/20 Periokuläres Basalzellkarzinom

03/20 Maximalinvasive Vorder-

abschnittschirurgie im Kindesalter 04/20 Qualitätssicherung bei der IVOM 05/20 Der Femtosekundenlaser in der Kataraktchirurgie 06/20 Personalisierte Medizin in der Behandlung von Augentumoren 07/20 SARS-CoV-2-Infektion und Auge 08/20 Space-Eye-Disease: Weltall, Auge und Glaukom

09/20 Retinologische Notfälle - das richtige Timing

10/20 Künstliche Intelligenz in der Augenheilkunde

11/20 Vergleichende Gegenüberstellung von Seh- und Hörbahn

12/20 Mikrochirurgische Operationstechniken aus ophthalmopathologischer Sicht

(Änderungen vorbehalten)

Alle Inhalte der Zeitschrift finden Sie unter www.springermedizin.de/ der-ophthalmologe 